\title{
ERRATUM: PIECEWISE CONSTANT COLLOCATION FOR FIRST-KIND BOUNDARY INTEGRAL EQUATIONS
}

\author{
I. G. GRAHAM ${ }^{1}$ and Y. YAN ${ }^{2}$
}

(Received 30 July 1993)

We wish to correct a minor error in the recent paper [2]. That paper was concerned with an integral equation defined on a closed polygon $\Gamma$ with $r$ corners at the points $\mathbf{x}_{0}, \mathbf{x}_{2}, \ldots, \mathbf{x}_{2 r}=\mathbf{x}_{0}$. We parameterized $\Gamma$ using a mapping $\gamma:[-\pi, \pi] \rightarrow \Gamma$ defined as follows. For each $\ell$, introduce the mid-point $\mathbf{x}_{2 \ell-1}$ of the side joining $\mathbf{x}_{2 \ell-2}$ to $\mathbf{x}_{2 \ell}$. Then introduce $2 r+1$ points in parameter space

$$
-\pi=S_{0}<S_{1}<\ldots<S_{2 r}=\pi
$$

with the property that for each $j=1, \ldots, 2 r$

$$
\left(S_{j}-S_{j-1}\right) / 2 \pi=m_{j} / M
$$

where $m_{j}$ are integers and $\sum_{j=1}^{2 r} m_{j}=M$. Then $\gamma(s)$ is defined by

$$
\gamma(s)=\mathbf{x}_{j-1}+\frac{\left(s-S_{j-1}\right)}{\left(S_{j}-S_{j-1}\right)}\left(\mathbf{x}_{j}-\mathbf{x}_{j-1}\right), \quad s \in\left[S_{j-1}, S_{j}\right],
$$

for $j=1, \ldots, 2 r$. The $\left\{S_{j}\right\}$ are then the preimages of the $\left\{\mathbf{x}_{j}\right\}$ under $\gamma$. Moreover, in view of (1), a family of uniform meshes can be constructed on $[-\pi, \pi]$ which include $\left\{S_{j}\right\}$ as the break-points. Then $\gamma$ maps these to meshes which are uniform on each segment joining $\mathbf{x}_{j-1}$ to $\mathbf{x}_{j}$ (which we denote $\Gamma_{j}$ ). These meshes are used to discretize the integral equation.

The map $\gamma$ is continuous but is not $\mathcal{C}^{1}$. In fact if $\hat{\mathbf{y}}_{j}$ denotes the unit vector in the direction $\mathbf{x}_{j}-\mathbf{x}_{j-1}$ then

$$
\gamma^{\prime}(s)=\left[\left|\Gamma_{j}\right| /\left(S_{j}-S_{j-1}\right)\right] \hat{\mathbf{y}}_{j}, \quad s \in\left[S_{j-1}, S_{j}\right] .
$$

\footnotetext{
'School of Mathematical Sciences, University of Bath, Claverton Down, Bath BA2 7AY, UK. ${ }^{2}$ Department of Mathematics, University of Kentucky, Lexington, KY 40506, USA.

(C) Australian Mathematical Society, 1994, Serial-fee code 0334-2700/94
} 
Clearly $\gamma^{\prime}$ is discontinuous at $S_{2 \ell}$ which is to be expected, since that is the preimage of a comer. However, our prescription also allows $\gamma^{\prime}$ to be discontinuous at $S_{2 \ell-1}$. (Note that, by definition, $\hat{\mathbf{y}}_{2 \ell-1}=\hat{\mathbf{y}}_{2 \ell}$ and $\left|\Gamma_{2 \ell-1}\right|=\left|\Gamma_{2 \ell}\right|$, but

$$
S_{2 \ell-1}-S_{2 \ell-2}=S_{2 \ell}-S_{2 \ell-1}
$$

was not assumed.)

As we show in the example below, if (4) is not true then operator $R$ discussed in Theorem 5 of [2] will have additional non-compact components besides those displayed there. The simple way to fix this problem is to require in addition that the integers $m_{j}$ satisfy

$$
m_{2 \ell-1}=m_{2 \ell}, \quad \text { for } \ell=1, \ldots, r .
$$

This ensures (4) and $\gamma^{\prime}$ becomes linear and hence $\mathcal{C}^{\infty}$ on each $\left(S_{2 \ell-2}, S_{2 \ell}\right)$. The ratios $d_{\ell}$ introduced in Section 3 may still be made arbitrarily close to 1 . To do this, let $p_{\ell} / q_{\ell}$ be a rational approximation to $\left|\Gamma_{2 \ell}\right|$ for $\ell=1, \ldots, r$. Then set $m_{2 \ell}=\left\{\prod_{j \neq \ell} q_{j}\right\} p_{\ell}$. Together with (5) this implies

$$
\frac{m_{2 \ell}}{m_{2 \ell+1}}=\frac{m_{2 \ell}}{m_{2 \ell+2}}=\frac{p_{\ell}}{q_{\ell}} / \frac{p_{\ell+1}}{q_{\ell+1}}
$$

which may be made as close to $\left|\Gamma_{2 \ell}\right| /\left|\Gamma_{2 \ell+1}\right|$ as desired.

Thus under the additional condition (5), all the results of the paper [2] remain correct.

EXAMPLE. Suppose $\mathbf{x}_{2 \ell-2}=(-1,0), \mathbf{x}_{2 \ell-1}=(0,0), \mathbf{x}_{2 \ell}=(1,0)$, and choose $S_{2 \ell-2}=-1, S_{2 \ell-1}=0, S_{2 \ell}=\alpha^{-1}<1$. Then consider the parametrization

$$
\gamma(s)= \begin{cases}(s, 0), & s \in[-1,0] \\ (\alpha s, 0), & s \in\left[0, \alpha^{-1}\right] .\end{cases}
$$

The operator $D(K-A)$ discussed in Theorem 5 of [2] has kernel

$$
k(s, \sigma)=-\frac{1}{\pi}\left\{\frac{\gamma^{\prime}(s) \cdot(\gamma(s)-\gamma(\sigma))}{|\gamma(s)-\gamma(\sigma)|^{2}}-\frac{1}{2} \cot \left(\frac{s-\sigma}{2}\right)\right\} .
$$

For $s \in[-1,0)$ and $\sigma \in\left(0, \alpha^{-1}\right], \cot (s-\sigma) / 2$ may be written as $2 /(s-\sigma)$ plus a smooth function of $(s, \sigma) \in[-1,0] \times\left[0, \alpha^{-1}\right]$. Hence using this and (6) 
we get, for $(s, \sigma) \in[-1,0) \times\left(0, \alpha^{-1}\right]$,

$$
\begin{aligned}
k(s, \sigma) & =-\frac{1}{\pi}\left\{\frac{1}{s-\alpha \sigma}-\frac{1}{s-\sigma}\right\} \\
& =-\frac{\alpha-1}{\pi}\left\{(\alpha-s / \sigma)^{-1}(1-s / \sigma)^{-1}\right\} \frac{1}{\sigma} .
\end{aligned}
$$

When $\alpha \neq 1$ this generates a non-compact operator on $H^{0}=L^{2}[-\pi, \pi]$.

Finally we remark that the results of [2] have been recently significantly generalised to the case of nonlinear parametrizations which yield smooth solutions of the integral equation. Optimal error estimates have been obtained for collocation methods of arbitrary high order applied to such equations in [1].

\section{Acknowledgement}

We thank Dr Johannes Elschner for many useful discussions.

\section{References}

[1] J. Elschner and I. G. Graham. "An optimal order collocation method for first kind boundary integral equations on polygons", submitted for publication.

[2] I. G. Graham and Y. Yan, "Piecewise-constant collocation for first-kind boundary integral equations", J. Austral. Math. Soc., Ser. B 33 (1991) 39-64. 